\title{
The Need for Clearance in Relaxation Therapy: Development of the Relaxation Clearance Tool for Practitioners
}

\section{Mavis Asare*and Samuel A Danquah}

Department of Psychology, Methodist University College Ghana, Ghana

*Corresponding author: Mavis Asare, Department of Psychology, Methodist University College Ghana, Ghana, Tel: +233272063193; E-mail: masare@mucg.edu.gh Received date: August 15, 2017; Accepted date: August 21, 2017; Published date: August 26, 2017

Copyright: (c) 2017 Asare M, et al. This is an open access article distributed under the terms of the Creative Commons Attribution License, which permits unrestricted use, distribution, and reproduction in any medium, provided the original author and source are credited.

\begin{abstract}
Relaxation therapy is one of the widely used therapies conducted in clinical psychology practice. The purpose of relaxation is to reduce tension in the various muscles in the body system. Relaxation therapy is considered a noninvasive intervention and is effective in treating a number of stress-related conditions. Both young people and adults benefit from relaxation therapies. Though this intervention is non-invasive, there is a need for proper screening of patients especially elderly clients and people with special conditions before relaxation therapies are conducted. Failure to screen individuals may result in some complications for the client in treatment. A clinical tool has therefore been developed to guide and help practitioners adequately screen their clients for relaxation therapy. At the end of the tool is an agreement section to be signed by the client and therapist before the relaxation therapy procedure.
\end{abstract}

Keywords: Relaxation clearance form; Relaxation therapy; Relaxation training; Behaviour therapy; Clinical psychology interventions

\section{Background}

Most frequently, some of the physical conditions presented by clients have stress-related component [1-3] which may need psychological intervention. A number of medical conditions have stress as an underlying factor [1-4] and in this case, the stress-related conditions need psychological intervention in order to promote a successful treatment. Relaxation therapy is one of the popular intervention procedures for stress disorders [2,5-7]. Stress is a psychological condition in which the person feels that the psychological demands are greater than the individual's ability to deal with these demands [8,9]. Symptoms of stress include persistent heartbeat, perspiration, continuous negative thoughts, etc. [5,6]. A stress condition begins at the cognitive level and later on affects the physiology of the person [10,11]. As stress affects the bodily process of the person, relaxation therapy is utilized in the intervention of stressrelated conditions $[5,6]$. There are various kinds of relaxation therapy and the most frequently used relaxation therapies for stress related conditions are the Progressive Muscle Relaxation, Autogenic and Biofeedback methods $[5,6]$.

Relaxation therapy is embedded in the behavioural theory of psychology $[7,12]$. Psychological treatment involves the transformation of these scientific theories into treatment procedures [5]. Relaxation therapy utilizes the principles of classical conditioning by the Physiologist Ivan Pavlov. A dog salivates when it is presented a piece of meat (unconditioned stimulus). The experimenter rings a bell (neutral stimulus) and shows the meat (unconditioned stimulus). Eventually, the dog salivates to the bell (conditioned stimulus) [12]. In relaxation training, an unconditioned physiological activation of tension is associated with un-tensing of muscles. This association is registered in the limbic system of the brain [13]. The body system eventually relaxes when there is a physiological trigger via the cognitive domain.
Relaxation therapy is one of the popular psychological treatments for the intervention of psychological disorders. Some of the conditions that have been successfully treated by relaxation therapy include anxiety, stress-related conditions and behaviour problems. Relaxation therapy is successfully utilised by both young people and adults.

\section{The Need for Screening before a Relaxation Therapy}

Relaxation intervention is considered a non-invasive procedure and WHO recommends psychological treatment involving the noninvasive procedures for the treatment of less severe mental health difficulties [14]. Although treatment involving relaxation is noninvasive with little or no side-effects $[2,5,6]$, it is necessary to screen clients before they undergo relaxation therapy. Screening before relaxation therapy is very important because some medical $[15,16]$ and mental conditions suffered by the client may prevent him/her from safely going through some aspects of the Relaxation Treatment Procedure [17-19]. Screening before relaxation therapy is therefore very necessary. The Progressive Muscle Relaxation involves holding in of breaths and tensing up of muscle groups $[5,6]$. For example: Clinical Observations indicated that 25 of 30 patients undergoing Progressive Muscle Relaxation were unable to hold on to their breaths for prolonged seconds (20 seconds). In the study, clients were instructed to stop when they felt uncomfortable [17]. Screening before the relaxation therapy helps the clinician to decide on the kind of relaxation procedure suitable for the client. This relaxation screening is particularly important when dealing with elderly clients in order not to complicate their already existing health conditions. Clients must complete the screening form before relaxation treatment begins.

\section{Importance of Relaxation therapy}

Most Stress conditions begin at the cognitive domain of the body system $[9,10]$. Stress begins as worries, negative thoughts and uncertainty in the brain. Stress therefore arises when the individual perceives the psychological and environmental demands as greater than capabilities to deal with those pressures. This means that stress originates from the cognitive state of a person and if not intervened, 
Page 2 of 4

this cognitive domain elicits hormones and neurotransmitters which affect the body system $[8-10,13]$. During relaxation treatment the following physiological changes take place [6].

- Body and mind typically enter a state of deep rest.

- Oxygen consumption can be lowered during 20-30 minutes relaxation to a point normally reached only after 6-7 hours of sleep.

- Heart rates typically decrease during relaxation.

- Forearm blood flow and forehead skin temperatures increase, indicating a dominance of the parasympathetic system.

- Electrical resistance of the skin also tends to increase significantly during relaxation indicating a lowering of anxiety at this time, while

- An excessive decline in the concentration of blood lactate may occur.

- The EEG (electroencephalograph) shows an alert-drowsy pattern with high alpha and occasional theta wave patterns as well as unusual pattern of shifts from alpha to slower (more sleep-like) frequencies.

\section{Kinds of Relaxation Therapy}

There are various kinds of relaxation procedures. The commonly utilised relaxation procedures in clinical psychological practice as already mentioned are described below:

Progressive Muscle Relaxation: The client tenses various muscle groups of the system $[5,6]$.

Autogenic Relaxation: Utilises mental images; following various pleasant mental scenes [5-7].

Biofeeback Relaxation Training: Measures physiological responses. Client becomes aware of his/her physiological arousal such as increased heart rate and then learn to cognitively control these abnormal physiological responses by stopping the cognitive triggers to the observed stress responses $[5,6]$.

\section{Medical and Mental Health Conditions Likely to be Incompatible with the Relaxation Procedures}

The following medical and mental health conditions may have system indications that may not be compatible with relaxation treatment procedures.

\section{Cardiovascular conditions}

People with cardiovascular conditions have hardening of the blood vessels, veins and arteries of the heart. This causes the heart muscles to become weaker. Holding on breaths may cause pains in the chest area and raise the blood pressure [15,16]. People with cardiovascular conditions should not perform Progressive Muscle relaxation which involves holding on breaths.

\section{Diabetes}

Diabetes condition is a malfunction of insulin. The person with this condition may not have stabilised sugar levels if the condition is not medically managed well $[15,16]$. Diabetes is also one of the biomarkers of a cardiovascular disease $[15,16]$. Sugar levels may go low and might not support the strong tensing of muscle groups as in Progressive Muscle Relaxation [15,16].

\section{Asthma}

People with asthma have blockage of airways [16]. Holding on breaths can interfere with their subsequent normal breathings.

\section{Elderly Persons}

Most of the cardiovascular conditions are seen among elderly people as a result of increased age. Older people above 55 years are more likely to suffer from high blood pressure and decreased aerobic capacity. These indications may limit their capacity for breath holdings $[15,16]$.

\section{Schizophrenia and Psychosis}

Persons with schizophrenia or psychosis experience hallucinations and delusions [18,19]. Their thinking and imagination can be distorted. They may for example experience bizarre mental images (hallucinations) and make bizarre meanings (delusions) to these images. Patients with schizophrenic conditions and psychosis are likely to distort the images and situations they imagine during Autogenic relaxation. In a clinical study of Autogenic training, patients attached bizarre meanings to mental images [15].

\section{Depression}

People with depression has low mood and they feel very dull $[18,19]$. Physical activities are one of the behavioural treatments for depression $[19,20]$. Depression is treated by providing stimulating environment with active scenes to raise the person's mood. Also, depressed people have low cognitive function [19,20]. Autogenic relaxation involves a solemn procedure where the client follows instructed mental images [5-7]. Patients with depression condition are likely to feel more depressed when going through non-active treatment procedures [17].

\section{Clinical Research Reports}

Clinical Observations indicated that 25 of 30 patients undergoing Progressive Muscle Relaxation were unable to hold on their breaths for prolonged seconds ( 20 seconds). 20 of 30 elderly patients (age range 55-60 years) had difficulties holding their breaths for 15 seconds. In this study, clients were instructed to stop the breath holding when they felt uncomfortable. Also, 36 of 40 patients with depression (age range 23-50 years) reported negative feelings after going through Autogenic Relaxation. 21 of 30 patients could not completely perform Autogenic relaxation with their Clinician [16].

\section{Methods}

\section{Description of the relaxation screening form (attached-form I \& II)}

The form consists of 13 items. These items are indications of cardiovascular and medical conditions which might prevent the individual from safely going through the relaxation procedure.

Chest pains, shortness of breath, palpitations, and hyperventilation are indicators of a cardiovascular condition including high blood pressure; dizziness is an indication of diabetes and anaemia (low blood levels). People with severe anaemia are likely to faint when tensing muscle groups $[15,16]$. 
Page 3 of 4

\section{Instruction}

The Clinician reads out the indicated symptoms to the client and the client responds by mentioning whether or not he/she experiences the symptoms at present or for the past weeks. If the client does not have the symptom a 'No' response is 0 . If the client has the symptom/ condition, the client rates the severity of the symptom indicated. The rating ranges from $1-3 ; 1=$ mild, $2=$ moderate and $3=$ severe. The clinician checks the client's responses on the Relaxation Screening Form. It takes an average of 10 minutes to complete the screening form. The clinician and the client sign at the end of the screening form.

\section{Scoring}

Total scores are not needed. It is scored by using the individual indicated symptom. Values are attached to the responses to indicate the severity of the symptom. A 'No' response is $0 ; 1=$ mild; $2=$ moderate; and $3=$ severe symptoms.

\section{Interpretation}

This is a screening tool; therefore the individual indicated symptoms are assessed to guide the clinician's decision on the type of relaxation procedure. The screening form is therefore interpreted based on each item indicated on the form.

Clients with 'NO' responses to all the indicated symptoms on the Relaxation Screening Form are cleared for all kinds of relaxation procedures. A client who responds 'Yes' but mild symptom (a rating of 1) for any of the symptom indicated on the form, can go through the relaxation procedure but under strict supervision. A client who responds 'Yes' with a rating of 2 or 3, indicating moderate to severe symptoms, to any of the items on the form, the relaxation procedure MUST be modified to suit the clients medical condition. The modification guide is attached on the Relaxation Screening Form.

\section{Validity and reliability of the relaxation screening form}

\section{Sample}

The screening form was tested with 160 clients attending the psychological health centre of Valley View Clinic and the Progressive Life Center. Informed consent was obtained from these clients. Clients' ages ranged from 35 to 65 years. The gender distribution was 98 (61\%) males and $62(38 \%)$ females. Of the 160 clients involved, 80 were previous clients who did not use the Relaxation Screening Form, and 80 were clients obtained for a period of 1 year who used the Relaxation Screening Form before relaxation treatment. The previous clients were those clients who were seen at the clinics before the development of the Relaxation Screening form. These two groups of clients, those previous clients who did not use the Screening form and those who used the Screening form, were assessed on complaints after Relaxation therapy.

\section{Assessment of complaints after relaxation therapy}

The complaints after Relaxation Therapy were assessed via verbal reports about feelings after relaxation therapies. These complaints have been documented in the clients' folders. The clients' complaints were quantified by the number of reported side effects after relaxation therapy. Clients who reported 'No complaints/side effect' is quantified as 0 , 'one side effect' $=1$, 'two side effects' $=2$, etc. The number of complaints indicates the total score on the Clients Complaint form.

\section{Results}

\section{Validity analysis}

The sample included 80 clients who used the Relaxation Screening Form.

The symptoms indicated on the Relaxation Screening Form correlated significantly with Physician's Diagnosis of the Client $(\mathrm{r}=0.65, \mathrm{p}<0.01)$.

\section{Reliability analysis}

The sample included 160 clients, 80 clients who were screened before Relaxation therapy and 80 who were not screened before Relaxation. Clients who were not screened before relaxation therapy reported significantly higher complaints after relaxation therapy $(\mathrm{M}=0.80, \mathrm{SD}=0.81)$ compared with clients who were screened before relaxation therapy $(\mathrm{M}=0.36, \mathrm{SD}=0.57 ; \mathrm{t} \quad(142)=3.906, \mathrm{p}<0.01)$. The magnitude of the differences in the mean values was very high (eta squared=0.08) [21]. Also, a Cronbach's Alpha value of 0.72 was determined for the Clients' reported symptoms and Physicians diagnoses of the client. This indicates a moderate to higher reliability of the Relaxation Screening Form [21].

\section{Discussion}

The Relaxation Screening Form has high validity and reliability. It is quick to use, and easily understood by the respondents. Indicated symptoms on the Relaxation Screening Form correlate with the Physician's diagnoses in the clients' hospital records/the Referral form by the Physician. When the Health Practitioner is considering a relaxation treatment procedure, the screening should be conducted to help the Clinician decide on the appropriate option suitable for the client. As shown in the text of this paper, some conditions suffered by clients have indications which are not compatible with relaxation procedures. A client with a medical condition can undergo relaxation therapy provided they are closely monitored. A client with a cardiovascular condition can perform a progressive muscle relaxation, only that some aspects of the procedure involving breath holding should be removed to suit the client's medical condition. Clinical Observations indicated that 25 of 30 patients undergoing Progressive Muscle Relaxation were unable to hold on to their breaths for prolonged seconds (20 seconds). 20 out of 30 elderly patients had difficulties holding their breaths for 15 seconds. Clients with Asthma should be closely supervised during relaxation procedures. They should have their inhalers close by during relaxation procedures. Diabetes is a condition of unstable sugar levels. When a client's diabetic condition is not properly managed, the relaxation procedure involving tensing of muscles should be closely supervised, or an alternative relaxation procedure such as Autogenic relaxation can be performed. Elderly clients should be properly screened as they are likely to suffer from numerous medical conditions associated with advanced age. Elderly clients are more likely to suffer from cardiovascular conditions, such as high blood pressure [14].

Clinicians must clear patients, especially elderly clients, before relaxation intervention. Clients whose conditions are not compatible with any of the relaxation procedures can undergo psychological interventions such as Cognitive Behaviour Therapy instead of relaxation treatment. 
Citation: Asare M, Danquah SA (2017) The Need for Clearance in Relaxation Therapy: Development of the Relaxation Clearance Tool for Practitioners. J Gen Pract (Los Angel) 5: 324. doi:10.4172/2329-9126.1000324

Page 4 of 4

\section{Conclusion and Recommendations for Health Practitioners}

Medical and mental health conditions may have system indications that may not be compatible with relaxation treatment procedures. Completing a screening form before relaxation is very important in clinical practice. The relaxation treatment procedure can be modified to suit the client's health condition or an alternative psychological intervention should be utilised.

Patients must be screened before beginning a relaxation therapy procedure. Clients with high blood pressure/taking blood pressure medications should not perform Progressive Muscle Relaxation. Clinicians can select an alternative relaxation procedure which does not involve tensing of muscles and holding in of breaths.

- Patients with diabetic condition should ONLY proceed with Progressive Muscle Relaxation when sugar levels are stabilised.

- Patients with Asthma should NOT perform Progressive Muscle Relaxation unless they have their inhalers with them.

- Patients with Schizophrenic conditions involving hallucinations should NOT perform Autogenic Relaxation.

- Patients with depression should NOT perform Autogenic relaxation.

- The clinician and the Patient/Client should sign the Relaxation Clearance Form in order to show responsibility from both parties.

\section{Acknowledgement}

The authors acknowledge the following Research Assistants: Lilian Ohene, Georgina Owusua Bonsu Bediako and Michellene Panni.

\section{References}

1. Schnelderman N, Ironson G, Siegel SD (2005) Stress and health Psychological, behavioural and biological determinants. Annu Rev Clin Psychol 1: 607-628.

2. Daskalopoulou S, Rabi DM, Zarnke KB, Dasgupta K, Nerenberg K, et al. (2015) The 2015 Canadian hypertension education program recommendations for blood pressure measurement, diagnosis, assessment of risk, prevention, and treatment of hypertension. Can J Cardiol 31: 549-568.
3. Progressive Life Center (2016) Annual Review on Clinical Practice. PLC, Accra, Ghana.

4. Plante TG (2005) Contemporary clinical psychology. (2nd Edn), John Wiley and Sons, New York, USA.

5. Walen S, Hauserman NM, Lavin PJ (1977) Clinical guide to behaviour therapy. Williams \& Wilkens Company, Baltimore, USA.

6. Davis M, Eshelman ER, McKay M (1988) The relaxation and stress reduction workbook. (3rd Edn), New Harbinger Publications, Oakland, USA.

7. Luthe W (1963) Autogenic training: Method, research and application in medicine. American Journal of Psychotherapy 17: 174-95.

8. Lazarus AA (1971) Behaviour therapy and beyond. McGraw Hill, New York, USA.

9. Selye H (1950). The physiology and pathology of exposure to stress.

10. Cannon WB (1929). Bodily changes in pain, hunger, fear and rage. (2nd ed), Appleton, New York, USA.

11. Meichenbaum D, Cameron R (1974) Modifying what clients say to themselves. In self-control: Power to the person by Mahoney MJ and Thoresen CE. Brooks/Cole, California, USA.

12. Anthony DJ (2003). Psychotherapies in counselling. Anugraha Publications, Bangalore, India.

13. Fincher J (1984) The human body. The brain: Mystery of matter and mind. Torstar Books Inc, New York, USA.

14. World Health Organisation (2015) WHO guidelines on conditions specifically related to stress.

15. Cojocaru E, Mastaleru A, Tamba B, Vasile R, Tudor RC, et al. (2017) Overview of some risk factors in cardiovascular disease. In: Kumar A (Ed), Recent trends in cardiovascular risks, InTech.

16. Insel PM, Roth WT (2004) Core concepts in health. McGraw-Hill, New York, USA.

17. Danquah SA, Asare M (2016) Clinical Research Reports. PLC, Accra, Ghana.

18. American psychiatric association (2013) Diagnostic and statistical manual of mental disorders- DSM-V. (5th edn), APA, Washington DC, USA.

19. Comer RJ (2007) Abnormal psychology. Worth Publishers, New York, USA.

20. Biddle SJH, Asare M (2011) Physical activity and mental health in children and adolescents: A review of reviews. Br J Sports Med 45: 886-895.

21. Field A (2009) Discovery statistics using SPSS. Sage Publications Ltd, London. 